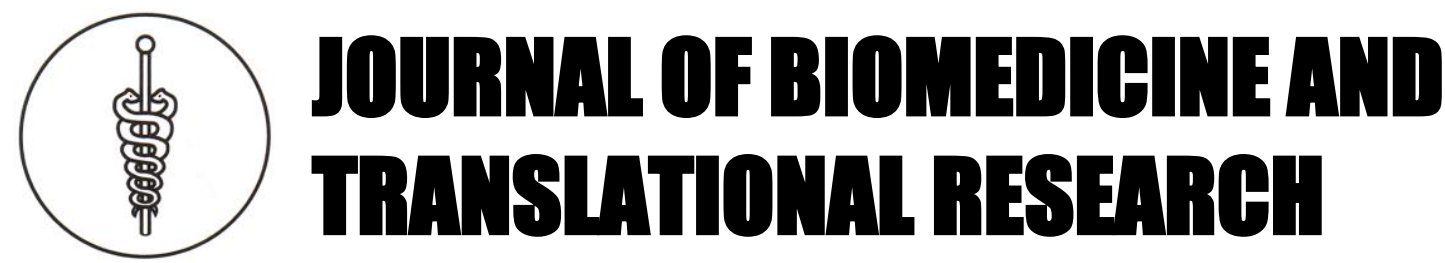

Copyright@2015 by Faculty of Medicine Diponegoro University and Indonesian Doctor Association, Central Java Region

\title{
Profile of Hypospadias Cases in Central Java, Indonesia
}

\author{
Ziske Maritska ${ }^{1}$, Ardy Santosa ${ }^{2}$, Mahayu Dewi Ariani ${ }^{1,3}$, Achmad Zulfa Juniarto ${ }^{1,3}$, Sultana \\ MH Faradz ${ }^{1}$ \\ ${ }^{1}$ Division of Human Genetics, Center for Biomedical Research, Faculty of Medicine Diponegoro University \\ ${ }^{2}$ Department of Urology, Dr. Kariadi Hospital \\ ${ }^{3}$ Medical Biology Department, Faculty of Medicine Diponegoro University
}

\section{Article info}

History:

Received 2 March 2015

Accepted 1 April 2015

Available 28 August 2015

\begin{abstract}
Background: Hypospadia is believed to be a multifactorial disease. The risk factors that may induce the formation of hypospadias are environmental factors, endocrine disruptors, and genetic factors. The aim of this study was to describe the profile of hypospadias patients who visited the Center for Biomedical Research (CEBIOR), Faculty of Medicine, Diponegoro University, Semarang, Indonesia.

Methods: This study is an observational study, using patients' medical record in CEBIOR from 2010 to 2012. The hypospadias cases were classified into syndromic, isolated and severe hypospadias based on their phenotype. The history of pregnancy, birth characteristics, and family history of the patients were described.

Results: There were 120 cases of hypospadias, consisted of $48.33 \%$ severe hypospadias, $41.67 \%$ mild isolated hypospadias and 10\% syndromic hypospadias. There were $38.33 \%$ hypospadias cases whose mothers were being exposed to repellant usage and $39.17 \%$ cases whose fathers were smoking. Forty (33.33\%) probands' mothers were aged above 35 years old when they gave birth to their affected son.

Conclusion: Majority of hypospadias cases were severe and mild isolated. Environtmental factors including maternal age more than 35 years old, use of repellant, and smoking fathers were found in this study.
\end{abstract}

Key Words: Hypospadias, profile, Indonesia

\section{INTRODUCTION}

Hypospadia is being considered as one of the most commonly found congenital anomalies, whereas it is also one of the most frequent male genital malformation ${ }^{(1,2)}$. The word "hypospadias" is originally derived from two Greek words, which are hypo and spadon. Hypo means below and spadon means hole, crack or gutter ${ }^{3-}$ ${ }^{5)}$. Hypospadias is defined as a condition where urethral meatus is ectopically located from its normal site ${ }^{(3)}$. The urethral meatus can be located anywhere along the urethral groove. It can be found at the tip of the glans penis, glanular, scrotal, or even perineal ${ }^{(3)}$.
There were various reports in number of hypospadias incidence worldwide. These differences might have been resulted from combination of many factors such as geographical, genetic, environmental influences, and also the variety of data collection ${ }^{(3)}$. Hypospadias is reported to occur about 1 out of 200-300 live male births in US population, while the incidence of hypospadias in European countries is around 3 per 1000 live male births ${ }^{(2)}$.

Hypospadias can be classified into few subtypes based on the position of the urethral meatus. The most commonly used classification of hypospadias was introduced by Duckett in 1996. He classified hypospadias into three groups based on the location of 
urethral meatus, i.e. anterior, middle and posterior form ${ }^{(5-7)}$. Hypospadias can also be classified into syndromic hypospadias and non-syndromic hypospadias. Syndromic hypospadias is a condition where hypospadias is a part of general syndrome. Example of syndromes which have hypospadias as one of their clinical features are Reifenstein, Wolf-Hirschorn, de Lange, and Fraser syndrome (3). Meanwhile, nonsyndromic or isolated hypospadias is a condition which hypospadias is the solely main clinical finding in a patient, without other symptoms found. Unlike syndromic hypospadias which has a clear cause, the underlying cause of non-syndromic hypospadias in most cases remains unknown ${ }^{(6)}$.

Although hypospadias is thought to be a multi factorial disease with elusive etiology, there are soome risk factors that can be associated with the occurrence of hypospadias. Some of the possible risk factors included environmental factors, endocrine disruptors, genetic factors, and other miscellaneous factors such as multiple birth, small gestational age, maternal age, smoking parents ${ }^{(1,9)}$.

Because of the vast possible risk factors for hypospadias, the aim of this study was to determine several environment and endocrine disruptors as the possible risk factors for hypospadias in male patients who visited CEBIOR, Faculty of Medicine, Diponegoro University, Semarang, Indonesia.

\section{MATERIAL AND METHODS}

This study used patients' data recorded at the Center for Biomedical Research (CEBIOR), Faculty of Medicine, Diponegoro University, Semarang, Central Java. All individuals with hypospadias who visited CEBIOR from 2010 to 2012 were included. All the patients had been examined thoroughly and been clinically diagnosed as hypospadias by the Disorders of Sexual Development (DSD) Team of CEBIOR. Pregnancy history, birth characteristics, and family history were obtained from the patients' parents or the patients themselves during their visit to CEBIOR. The pregnancy history included history of antenatal bleeding (ANB), drug usage during pregnancy, and smoking fathers. Whereas the birth characteristics been collected were weeks of gestation and birth weight. Consanguinity history in the family and the presence of other family members suffered from hypospadias were being asked as well. In addition, maternal age was also being included as one of the information being sought in this study.

There were a total number of 122 hypospadias cases during year 2010-2012 who visited CEBIOR, Semarang, Indonesia. However, two cases were excluded due to incomplete data record, leaving 120 total cases of hypospadias in this study. All the hypospadias cases were furthermore classified into three groups based on their phenotype and dysmorphologies found. They were severe, syndromic, and isolated hypospadias.

The severe hypospadias group is patients whose Quigley stage $\geq 3$, with other accompanying clinical features such as unpalpable gonad, bifid scrotum, and undescended testis. Meanwhile, the syndromic hypospadias group is hypospadias patients who had dysmorphologies found in their phenotype, other than hypospadias. The last group is the isolated hypospadias, in which hypospadias is their only symptom or clinical feature found, without other accompanying features.

\section{RESULTS}

There were 120 cases of hypospadias being included in this study, where 58 cases were severe hypospadias $(48.33 \%)$, followed by isolated hypospadias with 50 cases $(41.67 \%)$, and syndromic hypospadias with the least case found, which was only 12 cases (10\%). All the hypospadias cases in this study have had a chromosomal analysis, which were all 46, XY (100\%).

All the patients' mothers in this study had done the antenatal care (ANC) during their pregnancy (100\%). Some went to see general practitioners and/or midwives in the public health care; some even went to the obstetrician. During their pregnancy, there were nine patients' mothers $(7.5 \%)$ who had antenatal bleeding (ANB), five cases of severe hypospadias group, three cases of isolated hypospadias group, and one case of syndromic hypospadias.

As many as $86(71.67 \%)$ hypospadias patients from all groups have had history of environmental exposure, be it repellant usage, drug usage, chemical hazard, and smoking fathers, as displayed in figure 1 below.

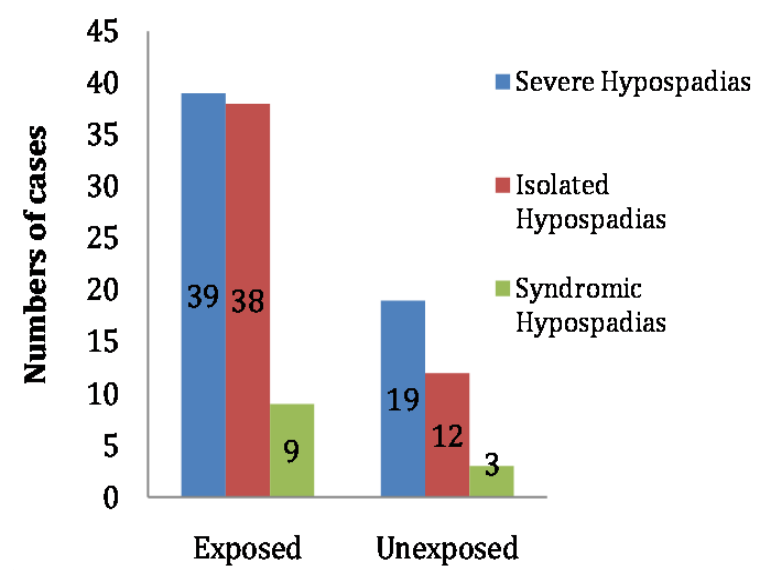

Figure 1. Environmental exposure history of hypospadias cases. Majority of all hypospadias patients from all groups $86(71.67 \%)$ have had history of environmental exposure. 
There are 46 cases $(38.33 \%)$ from all total cases where the parents were using repellant in various ways during pregnancy (24 cases of isolated hypospadias group, 21 cases of severe hypospadias group, and one case of syndromic hypospadias group). As many as 74 cases $(61.67 \%)$ told that the parents were not using any form of repellant during pregnancy, like it can be seen in figure 2 below.

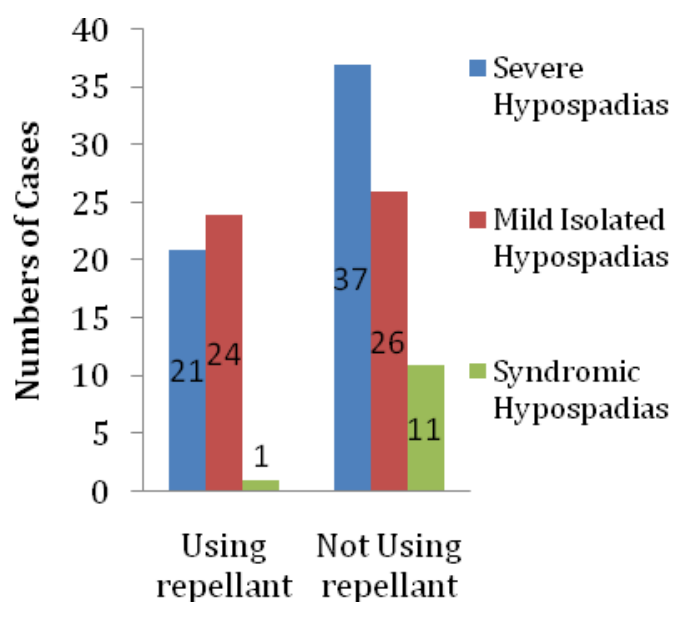

Figure 2. Repellant usage in hypospadias cases. A total of 46 hypospadias cases $(38.33 \%$ ) showed a positive history of repellant usage during pregnancy, while the other 74 cases $(61.67 \%)$ did not use repellant during pregnancy.

Regarding drug usage during pregnancy, most of the patients' mothers from all three groups only consumed vitamins and Fe supplement during their pregnancy $(n=88,73.33 \%)$. Only a few of them took other medicine with various indications. The second most commonly consumed drug in all hypospadias cases were drugs to strengthen pregnancy $(n=16$, $13.33 \%)$, followed by herbal medicine $(n=9,7.5 \%)$, flu drugs $(n=3,2.5 \%)$ and antibiotic and antiemetic as the least drug consumed, each with 2 patients $(1.67 \%)$.

In this study, most of the fathers of the patients stated that they have not been smokers $(n=73$, $60.83 \%$ ). However there were still quite a lot of participants whose fathers have been smokers $(n=47,39.17 \%)$, whom 26,19 , and 2 cases were in the severe, isolated, and syndromic hypospadias group respectively, as displayed in figure 3.

Endocrine disrupting chemicals are believed to be one of the underlying causes of hypospadias. Majority parents from all three groups stated that the wife did not have any contact or exposure with chemical hazard such as pesticide, insecticide, or other chemical compounds during pregnancy $(n=94$, $78.33 \%$ ). Only a few others who were exposed with chemical hazard, and the chemical hazard was mostly pesticide $(n=26,21.67 \%)$.

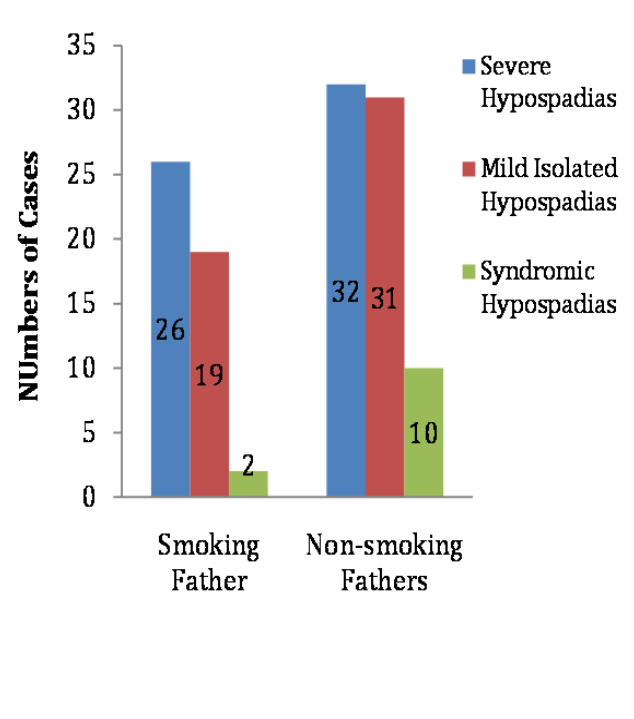

Figure 3. Smoking fathers of hypospadias patients. Most of the fathers were not smokers (73, $60.83 \%$ ), however 47 fathers $(39.17 \%)$ stated that they were a smoker.

Shorter period of gestation have somewhat been established as one of the possible risk factors for hypospadias. However, almost all patients in this study were born full term $(n=104,86.66 \%)$. Only 14 cases were born preterm $(11.67 \%)$ and the rest were even born post term $(n=2,1.67 \%)$

A low birth weight has been assumed to have association with the increased risk of hypospadias. In this study, the birth weight was classified into three groups, i.e: very low birth weight $(<1500$ gr), low birth weight (1500-2499 gr), normal birth weight (2500-4000 gr), and the group which the birth weight is more than 4000 gr. Most patients in all three groups were born with normal weight. There were 91 (75.83\%), 14(11.67\%), ans 4(3.33\%) patients who were born with a normal, low, very low, and more than normal birth weight respectively.

Regarding family history, almost all the patients did not have any consanguinity history $(n=118$, $98.33 \%)$. Nonetheless, there were two cases $(1.67 \%)$ that had consanguinity history. Both had severe and syndromic hypospadias. Moreover, there were 2 cases of hypospadias who had other family members affected (1.67\%); one case from syndromic 
hypospadias group and another from severe hypospadias group. The rest did not have any family member affected $(n+118,98.33 \%)$.

Maternal age is ranged based on the active reproductive age, which is 16-25 years old, 26-35 years old, and 36-45 years old. Most of the mothers were aged 26-35 years old when they had their affected $\operatorname{son}(n=69,57.5 \%)$, followed by age ranged from 36-45 years old or so called as the advanced maternal age with 40 mothers $(33.33 \%)$, and 11 mothers were aged around 16-25 years old, as shown on figutr 4 below.

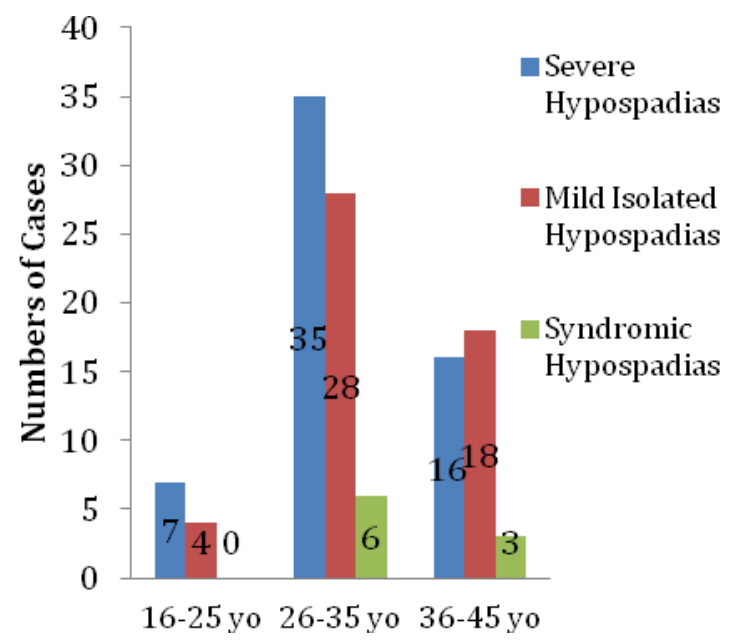

Figure 4. Maternal age of hypospadias patients. Majority of the mothers were aged around 26-35 y.o, $(n=69.57 .5 \%)$, followed by $36-45$ y.o. age group as many as 40 cases $(33.33 \%)$.

\section{DISCUSSION}

Hypospadias is widely regarded as a multi factorial disease, affected by many factors such as genetic and environmental factors. A study in Netherland found that the anterior and middle types of hypospadias were believed to be more affected by genetic factors ${ }^{(7)}$. Meanwhile, the posterior phenotype was more related with pregnancy risk factors, namely primiparity, preterm delivery, and being small for gestational age. In this study, more than half of patients in severe hypospadias group had a posterior type of hypospadias, where their orificum urethra externa was located at penoscrotal, while most of the syndromic hypospadias and isolated hypospadias cases had the anterior-middle form of hypospadias. Moreover, the hypospadias cases who were born preterm had various types of hypospadias based on the orificium urethra external location, i.e. anterior, middle, and posterior type. There was no tendency found that one type of hypospadias was related with preterm as its risk factors in this study.

One of the pregnancy history identified in this study was the presence of ANB during pregnancy, where there were only nine mothers $(7.5 \%)$ who had experienced of ANB during pregnancy. However, mothers who were having ANB during pregnancy were usually treated with some hormonal medication to stop the bleeding and subsequently, to prevent it from happening again in a certain period of time. The usage of these drugs may trigger the forming of hypospadias in their male offspring, but this assumption needs further study.

In a way to see whether some drugs are by chance can be possible pronounced risk factors in the developing of hypospadias, this study also asked about drug usage history during pregnancy to the mothers. There was one study conducted in Iran suggested that iron and folic acid supplementation treatment during first trimester may have preventive effect in hypospadias ${ }^{(10)}$. The second most common consumed drug in all the hypospadias cases were drugs to strengthen pregnancy, given with various indications. Progesterone and its derivatives were quite commonly being prescribed during early period of pregnancy, despite there was a report that consumption of progestins in early pregnancy may be associated with an increased risk of hypospadias (11)

Endocrine disruptors are believed to be one of the underlying etiologies of hypospadias. Some of the examples of potential endocrine disruptors are pesticides, fungicides, industrial chemical product, detergents, material fabrication of plastics, and even cigarette smoke. Humans can be exposed to these materials though many ways, such as diet, food packaging, or direct contact in the occupational setting. Some of the type of exposure found in this study was through the use of repellant, contact with pesticides, and cigarette smoke. There were only a few cases in this study that had been exposed to chemical hazard during pregnancy, whereas the chemical hazard mostly contacted were pesticide, due to their occupation as a farmer. Some studies did show an association between exposure of pesticides or other environmental chemical such as industrial and agricultural products with the incidence of hypospadias. Kim et al. found that incidence of hypospadias in Korea was higher in industrialized areas compared to the nonindustrialized areas ${ }^{(12)}$.

Although majority of the fathers stated that they were not smokers or did not smoke during 
pregnancy, there is still quite large number of patients' fathers who were smoking. There are studies showing that there is an association between the risk of hypospadias and exposure to passive smoking done by smoking fathers ${ }^{(13,14)}$. One of the mechanism of which exposure to passive smoking can induce hypospadias is smoking may cause a germ line mutation that might lead to the occurrence of hypospadias in the offspring ${ }^{(14)}$.

Period of gestation and birth weight are the two birth characteristics that mostly identified in a lot of previous researches. Based on a few studies, infants with hypospadias were more likely to be delivered at gestational age of $<37$ weeks ${ }^{(15,16)}$. Being said that, only 14 cases were born preterm in this study, where seven cases were the severe hypospadias group. A study in Italia proposed a theory that the incidence of hypospadias -especially the moderate and severe type- tends to be higher in infants born small for gestational age (SGA) than in newborns with appropriate gestational age (AGA) ${ }^{(16)}$. Low birth weight may reflect a placental insufficiency. Hence, there is a lack of nutrients and gonadotrophins needed for the development of the fetus ${ }^{(1)}$.

In siblings, boys with hypospadias have been shown to have lower birth weights compared to their healthy brothers ${ }^{(17)}$. People with hypospadias tend to have lower birth weight compared to their twins or siblings, suggesting a gene-environment interaction. Unfortunately, this study only recorded patients' birth weight, not including their siblings' birth weight either. However, only a small portion of cases that had low birth weight, where more than half of the cases had normal birth weight.

There are $10 \%$ cases of hypospadias which is a familial clustering (1). Familial clustering is described as patient having one or more, whether it is first, second, or third degree relatives that are also affected with hypospadias. In this study there were two cases of which they had other family members affected. All the cases are from severe hypospadias and syndromic hypospadias group, none from the isolated hypospadias group. One case in severe hypospadias group had his grandfather's little brother having the same condition as he is. While one case in syndromic hypospadias group had a first-degree relative affected, his younger brother. Moreover, parents of this sample are also a firstdegree cousin, means it is a consanguineous marriage. A case-control study in Netherland identified that the anterior and middle hypospadias seemed to have more family history compared to the posterior one ${ }^{(7)}$. In this study, those who have family history belong to syndromic hypospadias group and severe hypospadias group. One case had his urethral meatus located on distal penile, which is an anterior type of hypospadias, while the other one had his urethral meatus in penoscrotal, which is a posterior type of hypospadias.

A study conducted in Washington State showed that there were strong association between older maternal age and the increased risk of hypospadias among male offspring. Women older than 40 years old were at greatest risk of having son with hypospadias ${ }^{(15)}$. Study by Fisch et al. reported that women $>35$ years of age had a $50 \%$ higher risk of having male offspring with hypospadias compared with women $<20$ years of age in California, and had a $20 \%$ higher risk for the same group of age in New York ${ }^{(18)}$. This study found that there were 40 women $(33.33 \%)$ were aged above 35 years old when they gave birth to their affected son.

\section{CONCLUSIONS}

Majority of hypospadias cases in this study were severe and mild isolated. Environtmental factors including maternal age more than 35 years old, use of repellant, and smoking fathers were found among those cases.

\section{References}

1. Kalfa N, Philibert P, Sultan C. Is hypospadias a genetic, endocrine or environmental disease, or still an unexplained malformation? Int J Androl. 2009;32:187-197.

2. Caione P. Prevalence of hypospadias in European countries: Is it increasing? Eur Urol. 2009;55:1027-1029.

3. Leung AKC, Robson WL. Hypospadias: an update. Asian J Androl. 2007;9:16-22.

4. Nassar N, Bower C, Barker A. Increasing prevalence of hypospadias in Western Australia, 1980-2000. Arch Dis Child. 2007;92:580-584.

5. Michalakis M, Heretis G, Chrysos E. Tsatsakis A. Pesticides Exposure and Risk of Hypospadias. In: Stoytcheva M (editor). Pesticides-The impact of Pesticide Exposure. Cited from http://www.intechopen.com/books/pesticidesthe-impacts-of-pesticides-exposure/pesticidesexposure-and-risk-of-hypospadias on October 23, 2014.

6. Troncoso B, Lopez PJ. Hypospadias. Pediatric Urology Book. Cited from 
http://www.pediatricurologybook.com/hypospa dias.html on October 23, 2014

7. Van Rooij IA, Van der Zanden LF, Brouwers MM, Knoers NV, Feitz WF, Roeleveld N. Risk factors for different phenotypes of hypospadias: results from a Dutch case-control study. BJU Int. 2013;112:121-128.

8. Manson JM, Carr MC. Molecular epidemiology of hypospadias: review of genetic and environmental risk factors. Birth Defect Research (Part A). 2003;67:825-836.

9. Van der Zanden LF, Galesloot TE, Feitz WF, Brouwers MM, Shi M, Knoers NV, et al. Exploration of gene-environment interactions, maternal effects and parent origin effects in the etiology of hypospadias. J Urol. 2012;188:2354-2360.

10. Mohammadzadeh A, Farhat A, Esmaieli H, Shiranzaei S. Prevalence and risk factors of hypospadias in a private hospital In Northeast Iran. Iran J Pediatr. 2011;21: 497-501.

11. Charmichael SL, Shaw GM, Laurent C, Croughan MS, Olney RS, Lammer EJ: Maternal progestin intake and risk of hypospadias. Arch Pediatr Adolesc Med. 2005; 159:957-962.

12. Chul Kim S, Kyoung Kwon S, Pyo Hong Y. Trends in the incidence of cryptorchidism and hypospadias of registry-based data in korea: a comparison between industrialized areas of petrochemical estates and a non-industrialized area. Asian J Androl. 2011;13:715-718.

13. Akre O, Boyd HA, Ahlgren M, Wilbrand K, Westergaard T, Hjalgrim H, et al. Maternal and gestational risk factors for hypospadias. Environ Health Perspect. 2008;116:1071-1076.

14. Pierik FH, Burdorf A, Deddens JA, Juttmann RE, Weber RFA. Maternal and paternal risk factors for cryptorchidism and hypospadias: A case-control study in newborn boys. Environ Health Perspect. 2004;112:1570-1576.

15. Porter MP, Faizan MK, Grady RW, Mueller BA. Hypospadias in Washington state: Maternal risk factors and prevalence trends. Pediatrics. 2005;115:495-499.

16. Ghirri P, Scaramuzzo RT, Bertelloni S, Pardi D, Celandroni A, Cocchi G, et al. Prevalence of hypospadias in Italy according to severity, gestational age and birthweight: an epidemiological study. Ital J Pediatr. 2009;35:18.

17. Boisen KA, Chellakooty, Schmidt IM, Kai CM, Damgaard IN, Suomi AM, et al. Hypospadias in a cohort of 1072 Danish newborn boys: Prevalence and relationship to placental weight, anthropometrical measurements at birth, and reproductive hormone levels at three months of age. J Clin Endocrinol Metab. 2005;90:4041-4046.

18. Fisch H, Golden RJ, Libersen GL, Hyun GS, Madsen P, New MI, Hensle TW. Maternal age as a risk factor for hypospadias. J Urol. 2001;165:934-936. 